\title{
Variation in gut microbial communities and its association with pathogen infection in wild bumble bees (Bombus)
}

\author{
Daniel P Cariveau, J Elijah Powell, Hauke Koch, Rachael Winfree and Nancy A Moran
}

The ISME Journal (2014) 8, 2550-2551; doi:10.1038/ismej.2014.180

Correction to: The ISME Journal (2014) 8, 2369-2379; doi:10.1038/ismej.2014.68; published online 24 April 2014

Since the publication of this article, the authors have identified incorrectly presented results in their paper. These relate to the absolute numbers of bacteria present in the gut communities of the three host species.

Figure 5 showed means from model output rather than absolute numbers of $16 \mathrm{~S}$ rRNA copies as described in the legend and text.

The authors have redrawn Figure 5 with the actual means, corrected error bars and corrected y-axis label. As a result of this, the sentence (within the Results section) 'Results from the generalized linear mixed model indicated that the log copy number was highest in B. impatiens (14.2 \pm 0.35 s.e., Figure 5), followed by $B$. griseocollis (13.6 \pm 0.37 s.e., Figure 5) and

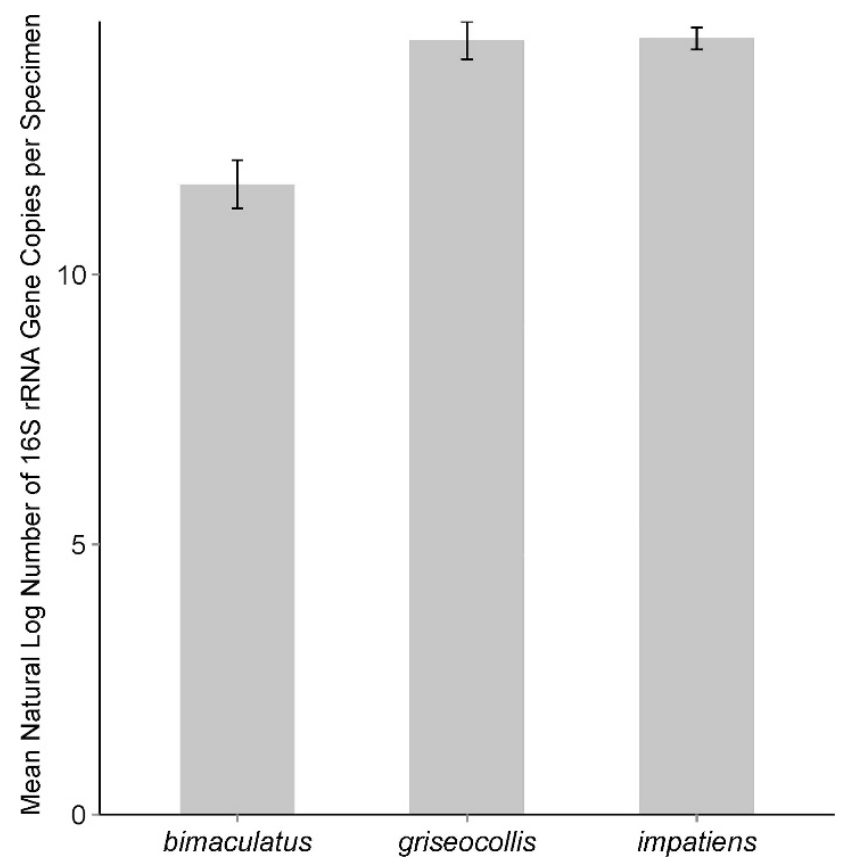

Figure 5 Means of natural log-transformed numbers of bacterial $16 S$ rRNA gene copies per specimen. Error bars represent \pm s.e.m.
B. bimaculatus $(12.7 \pm 0.41$ s.e., Figure 5)' should now read:

'Results from the generalized linear mixed model indicated that the log copy number was highest in $B$. impatiens (14.37 \pm 0.21 s.e., Figure 5), followed by $B$. griseocollis (14.3 \pm 0.35 s.e., Figure 5) and $B$. bimaculatus ( $11.7 \pm 0.45$ s.e., Figure 5$)$.'

Additionally, the y-axis of Figure 6 should also read 'Natural Log Number of $16 \mathrm{~S}$ rRNA Gene Copies per Specimen', as in Figure 5.

These issues do not affect the results of statistical analyses or the conclusions of the manuscript overall.

The complete data set used for this manuscript will be available online through Dryad Digital Repository (doi: 10.5061/dryad.3k019).

In addition, the authors have identified an error within Supplementary Table 6, namely that it contained an old analysis resulting in the Chao 1 being different from the means presented in the main text.

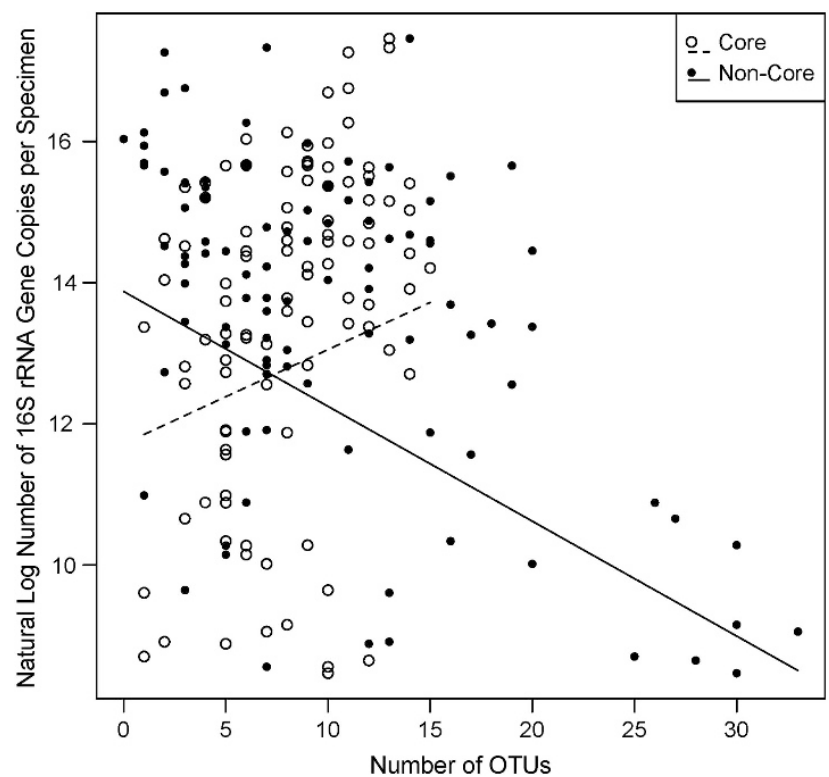

Figure 6 Results of mixed model analysis showing that the richness of non-core (closed circles and solid line) bacteria OTUs is negatively associated with total number of 16S rRNA gene copies per bee specimen, whereas the richness of core (open circles and dashed line) bacteria OTUs is positively associated. 
The main text, however, is correct (as are the supplementary figures), and this oversight does not change the conclusions of this analysis.

The errors have now been rectified, and the correct article appears in this issue. The html and online pdf versions have also been rectified, and now carry the correct paper (including the corrected Supplementary Table 6, which now presents the correct statistics for the $t$-tests and ANOVA).

The authors would like to apologise for any inconvenience this may have caused.

\section{Natural occurrence of microbial sulphur oxidation by long-range electron transport in the seafloor}

Sairah Y Malkin, Alexandra MF Rao, Dorina Seitaj, Diana Vasquez-Cardenas, Eva-Maria Zetsche, Silvia Hidalgo-Martinez, Henricus TS Boschker and Filip JR Meysman

The ISME Journal (2014) 8, 2551-2552. doi:10.1038/ismej.2014.170

Correction to: The ISME Journal (2014) 8, 1843-1854; doi:10.1038/ismej.2014.41; published online 27 March 2014

Since the publication of this article, the authors have identified an error within Figure 2, namely that two accession numbers for the clones in panel $\mathrm{d}$ (a phylogenetic tree) were incorrect.

The correct accession numbers are Aarhus (JX091070.1) and Aarhus (JX091067.1), and the correct figure is shown below.

The Authors would like to apologise for any inconvenience this may have caused. 